Revista Herencia Vol. 27 (1 y 2), 27-38, 2014

Recibido 19-09-2013 Aprobado 20-10-2013

\title{
RUTA HISTÓRICO-ARQUITECTÓNICA EN EL Cantón de Esparza, Puntarenas: propuesta para reforzar la asignatura de Educación Cívica del Ministerio de Educación Púlblica (MEP)
}

\begin{abstract}
Resumen
El presente trabajo aborda la realización de una ruta histórico-arquitectónica en el cantón de Esparza, Puntarenas, para reforzar la asignatura de Educación Cívica impartida por el Ministerio de Educación Pública (MEP). Se analizan diferentes aspectos implícitos como su viabilidad en el momento de ser utilizada por el docente.
\end{abstract}

Palabras claves: Educación Cívica, turismo arquitectónico, Esparza.

\begin{abstract}
The present article explains the development of a historical-architectural tour in Esparza, Puntarenas in order to strengthen Civics education contents of the Ministry of Public Education of Costa Rica. Analysis of different implicit aspects like its viability for being implemented will be taken into account.
\end{abstract}

Keywords: Civics Education, architectural tourism, Esparza.

\section{Introducción}

Carlos Eduardo Ledezma Benavidez.

Graduado en la carrera de Turismo Ecológico en la Sede del Pacífico, Universidad de Costa Rica. Actualmente estudiante de la Licenciatura en Gestión Ecoturística de la Universidad de Costa Rica, sede Guanacaste. alucar888@gmail.com
Al hablar de la actividad turística se hace hincapié en la cantidad de divisas que esta pueda generar y en la manera de reducir el impacto negativo que acarrea; pero estas ideas son muy lineales, pues la actividad turística es dinámica (como se ha observado en el tiempo), por su característica de adaptabilidad, lo que le permite complementarse con otras disciplinas.

Entonces, puede pensarse que desarrollar una ruta de turismo arquitectónico sería muy fácil si se cuenta con los atractivos culturales; no obstante, el reto de la presente propuesta radica en la elaboración de una ruta no convencional -ya que su objetivo principal no es generar divisas- y se centrará en la tarea de complementarse con otra disciplina para descubrir o revitalizar el conocimiento cultural de los jóvenes de una determinada área geográfica.

Cabe mencionar que esta propuesta debe seguir los lineamientos que caracterizan las actividades bajo el término turismo cultural, debido a que se va a trabajar con atractivos culturales-arquitectónicos. En este punto, es necesario definir dicho término como: 
... aquella forma de turismo que tiene por objeto, entre otros fines, el conocimiento de monumentos y sitios histórico-artísticos. Ejerce un efecto realmente positivo sobre éstos en la medida que, por sus propios medios, intervienen para mantenerlos con vida y protegerlos. Esta forma de turismo justifica, de hecho, los esfuerzos que tal mantenimiento y protección exigen de la comunidad humana, en razón de los beneficios socio-culturales y económicos que de allí se desprenden para toda la población implicada. (ICOMOS, 1976; citado por Alfaro y Porras, 2006. Tesis de licenciatura, inédita).

Siguiendo estos lineamientos, es posible desarrollar una ruta de turismo arquitectónica en el cantón de Esparza, Puntarenas. El cantón esparzano, dentro de sus límites territoriales, cuenta con ocho sitios declarados de interés históricoarquitectónicos, los cuales son parte importante de la identidad cultural de los esparzanos, conocidos popularmente como "garroberos".

Si bien esta ruta puede realizarse, por sí sola la meta propuesta no sería posible de alcanzar pues es necesaria la complementariedad de dos disciplinas: la turística, que proporciona un sinnúmero de estrategias para gestionar los atractivos culturales; y la asignatura de Educación Cívica del MEP, que proporciona, por su cuenta, la formación del "ser ciudadano" en los jóvenes e involucra rasgos culturales.

\section{Breve reseña histórica de la cuidad y sus monumentos arquitectónicos}

La primera vez que se menciona el nombre de "La Ciudad del Espíritu Santo" es en 1574; por esta razón, se considera que el cantón de Esparza es el segundo asentamiento en establecerse dentro del territorio nacional después de Cartago. El responsable de este primer asentamiento en Esparza fue el gobernador interino de la época don Alonso Anguciana de Gamboa, quien decidió trasladar la población de Aranjuez al valle de Coyoche, cerca del poblado de Artiada. No obstante, la palabra "Esparza" se le asignó tres años después, en 1577, por Diego Artieda y Chirino, en honor a la villa que lo vio nacer: Esparza, localizada en Pamplona, España, así que el nuevo nombre que adquiere la ciudad sería "Ciudad del Espíritu Santo de Esparza". Aunado a estos hechos, el segundo asentamiento -y último de la llamada Ciudad del Espíritu Santo de Esparza- se localizó a cinco kilómetros de su antigua posición y dicho cambio se presentó entre 1622 y 1629. (Sandi, 2003).

Uno de los acontecimientos más importantes, desde el punto de vista histórico -y que debe ser mencionado en el desarrollo del cantón "esparzano", ya que actualmente repercute en los ciudadanos-, es el cambio de nombre de la cuidad de Esparza por el de Esparta. Este cambio se registró el 3 de setiembre de 1879 por el General Tomás Guardia, mediante el decreto N. 93 (Lagos, 1976), el acuerdo decía lo siguiente:

Secretaría de Gobernación, Palacio Nacional, San José 3 de septiembre de 1879. Considerando que la ciudad de Esparza, según documentos antiguos, en otro tiempo se denominó Esparta y que sus principales han manifestado deseos de que recobre el nombre primitivo, el presidente acurda que, en lo sucesivo la expresada ciudad, en todos los documentos oficiales, vuelve a denominarse Esparta, Publíquese. Rubricado por S.E., el General presidente, (F) Manchado.

Sin embargo, para 1945, el doctor Jorge Volio Jiménez amplió la información de este suceso exponiéndolo como un error de la época, según lo describe a continuación: 
Haciendo destacar en nota que dicho cambio fue debido a manera grecomanía de la época y que, contrariamente a lo que afirma en este acuerdo, "no hubieran podido citar un sólo documento antiguo" donde se llamara Esparta a la cuidad del Espíritu Santo de Esparza [....] no hemos visto nunca la grafía "Esparta" ni siquiera por yerro de algún amamense distraído. A esa circunstancia que quería referir al calificar de "inexplicable" este cambio de nombre. (Lagos, 1976, pp. 12-14).

Es de suma importancia mencionar este hecho histórico, ya que desde esta fecha, 1879, la documentación que se encuentra sobre el cantón va a hacer referencia a la palabra "Esparta" y no a "Esparza".

Con este nuevo nombre, Esparta desarrolla un gran auge en lo referente a actividades sociales después de 1950, como por ejemplo: el regio té de modas en donde se hacían demostraciones de arreglos florales y de productos de belleza y números artísticos (La Nación, 1966). También se realizaban bailes en el Centro Cultural Recreativo Espartano para celebrar acontecimientos del pueblo o para recaudar fondos destinados a las diferentes instituciones. (La Nación, 1965).

Para 1974, se le devuelve el nombre de Esparza al cantón segundo de la provincia de Puntarenas, bajo el decreto N. ${ }^{\circ} 3752-G-C$ del 6 mayo (Sandi, 2003); pero

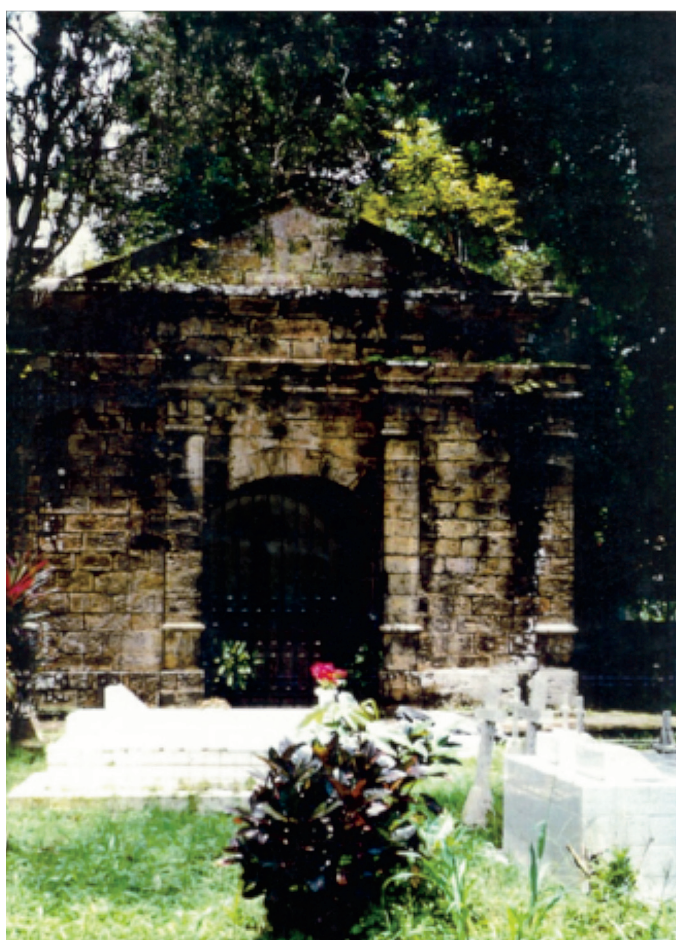

Figura 1. Capilla del cementerio de Esparza, construida por Juan Ona, en piedra canteada. Fuente: Marcos Hernán Elizondo. este hecho no tuvo gran trascendencia en el quehacer cotidiano de los pobladores, lo cual se refleja en la actualidad pues todavía la población costarricense utiliza expresiones como: "el centro de Esparta" o "la ciudad de Esparta", entre otros. Incluso, los mismos esparzanos utilizan ambas palabras como sinónimos.

A pesar de ser el segundo asentamiento español en establecerse en el territorio costarricense, se considera que los límites territoriales del cantón fueron zonas habitadas por grupos indígenas, entre ellos destacan: los chorotegas, que procedían de Orotina - para ese tiempo gobernados por el Cacique Guritiña-y los huetares - que procedían del occidente (Sandi, 2003)—, lo que le proporciona al territorio mayor riqueza histórica.

\section{Patrimonios histórico-arquitectónicos de Esparza}

Actualmente, la información que se encuentra sobre los monumentos arquitectónicos es muy escasa; sin embargo, algunos trabajos, como los realizados por el politólogo esparzano Marcos Hernán Elizondo Vargas, han permitido desarrollar breves descripciones de los acontecimientos más importantes del cantón e incluyen información acerca de los monumentos por tratar.

Don Hernán ha dedicado parte de su tiempo a la recolección de datos generales del cantón, presentes en los registros nacionales. Con su labor ha elaborado 8 tomos que se destacan por estar en orden cronológico y presentan un formato de agenda histórica. En dichos tomos se trascriben, sin alteraciones, los sucesos que se han presentado en el cantón. Estos tomos pueden ser consultados en la Biblioteca interna de la Municipalidad de Esparza y en la biblioteca personal de don Hernán Elizondo. 
Basándose en estos tomos, la Municipalidad de Esparza ha realizado breves reseñas históricas de los monumentos que pueden ser consultados en la página web oficial de la entidad, además existen varios blogs en Internet que presentan información muy similar.

Por lo anterior, es importante aclarar que, para el presente trabajo, se utilizará la información suministrada por la Municipalidad de Esparza y por la página web oficial del Centro de Investigación y Conservación del Patrimonio Cultural para, así, realizar una breve caracterización de los atractivos culturales con declaratoria de interés histórico-arquitectónico - dado que el objeto del trabajo no es profundizar en la historia de tales atractivos, ya que esto sería redundar en la información ya publicada-.

A continuación se describen los 8 monumentos arquitectónicos que conformarán la ruta turística y que serán el eje fundamental de la propuesta curricular de la asignatura de Educación Cívica del MEP (ver Cuadro 1).

\section{Cuadro 1}

\section{Descripción de los atractivos arquitectónicos de Esparza}

\begin{tabular}{|c|c|}
\hline Monumento & Descripción \\
\hline El puente de Las Damas & $\begin{array}{l}\text { Este puente es el primero en ser declarado como monumento histórico ar- } \\
\text { quitectónico del cantón, mediante Decreto Ejecutivo N. }{ }^{\circ} 4990-C ., \text { del } 23 \\
\text { de junio de } 1975 \text {. Se construyó sobre el río Jesús María, con piedra tallada } \\
\text { en el siglo XIX. Su nombre se origina en honor a las señoras y señoritas } \\
\text { cafetaleras y comerciantes que colaboraron con dinero para la obra, ac- } \\
\text { ción solicitada por Sociedad Económica Itineraria de Costa Rica. }\end{array}$ \\
\hline El cementerio de Esparza & $\begin{array}{l}\text { Declarado monumento patrimonial de interés histórico arquitectónico, me- } \\
\text { diante decreto N. }{ }^{\circ} 23061-C \text { de julio de } 1994 \text {. Haciendo énfasis en su periodo de } \\
\text { construcción que data del siglo XIX, para ser más exacto 1851, cuenta con una } \\
\text { capilla de piedra canteada y esculturas de mármol y piedra de gran belleza. }\end{array}$ \\
\hline $\begin{array}{l}\text { El parque Ignacio } \\
\text { Pérez Zamora }\end{array}$ & $\begin{array}{l}\text { Fue declarado de interés histórico-arquitectónico, según Decreto Ejecutivo } \\
\text { No } 21626-C \text {, publicado en La Gaceta N. } 213 \text { del } 5 \text { de noviembre de } 1992 \text {, de } \\
\text { carácter estatal. El parque fue conocido como una plaza de mercado hasta } \\
\text { finales del siglo XIX. Su construcción se empezó en } 1900 \text { y se inauguró el } 31 \text { de } \\
\text { diciembre del mismo año. En el centro se encuentra un quiosco de estilo neoclá- } \\
\text { sico que es una prolongación arquitectónica de la torre del templo católico. }\end{array}$ \\
\hline El túnel de Cambalache & $\begin{array}{l}\text { Declarado monumento patrimonial de interés histórico-arquitectónico, me- } \\
\text { diante decreto ejecutivo N. }{ }^{\circ} 24083-C \text {, de febrero de } 1995 \text {. Tiene una altura } \\
\text { aproximada de } 7 \text { metros y un ancho de unos } 4.5 \text { metros en la boca del túnel. } \\
\text { Su estilo constructivo es en arco y su extensión es de } 300 \text { metros de largo. }\end{array}$ \\
\hline Túnel de Miraflores & $\begin{array}{l}\text { Declarado patrimonio de interés histórico-arquitectónico, mediante decre- } \\
\text { to N. }{ }^{\circ} 24087-C \text {, publicado en La Gaceta N. }{ }^{\circ} 54 \text { del } 16 \text { de marzo de } 1995 . \text { Es } \\
\text { una construcción en piedra canteada (al menos en sus primeros } 20 \text { metros) } \\
\text { y, en sus dos entradas, es de roca viva. Este túnel, de } 210 \text { metros de largo, } \\
\text { ha de tener entre } 3.5 \text { y } 4 \text { metros de ancho por unos } 6.5 \text { metros de alto. }\end{array}$ \\
\hline
\end{tabular}




\begin{tabular}{|c|c|}
\hline Monumento & Descripción \\
\hline La estación de Caldera & $\begin{array}{l}\text { Declarada monumento patrimonial de interés histórico-arquitectónico me- } \\
\text { diante decreto N. } .^{\circ} 23978-C \text {, publicado en La Gaceta N. }{ }^{\circ} 33 \text { del } 15 \text { de febrero } \\
\text { de } 1995 . \text { La edificación presenta una estructura en concreto armado de dos } \text { niveles. En la planta baja, se localizan tres aposentos y, en la planta alta, se } \\
\text { ubican dos grandes salones, esta dejó de funcionar como tal en } 1989 . \text { Ac- } \\
\text { tualmente, se encuentra arrendada por el INCOFER (Instituto Costarricense } \\
\text { de Ferrocarriles) a una persona particular, quien la explota comercialmente. }\end{array}$ \\
\hline Escuela Arturo Torres Martínez & $\begin{array}{l}\text { Declarada monumento patrimonial de interés histórico-arquitectónico, } \\
\text { mediante decreto N. } 196-95-C \text { del } 18 \text { de abril de } 1990 \text {. Fue inaugura- } \\
\text { da en abril de } 1946 \text { y fue llamada así en honor a su fundador: Arturo } \\
\text { Torres Martínez. La construcción de la obra fue supervisada por el } \\
\text { arquitecto José María Barrantes, uno de los más destacados arquitec- } \\
\text { tos de edificaciones públicas y privadas de mediados del siglo XX. }\end{array}$ \\
\hline $\begin{array}{l}\text { La Casa de la Cultura Juan } \\
\text { Bautista Matamoros }\end{array}$ & $\begin{array}{l}\text { Declarada monumento patrimonial de interés histórico-arquitec- } \\
\text { tónico, mediante decreto N. }{ }^{\circ} 29358-C \text {, publicado en La Gaceta N. } \\
53 \text { del } 15 \text { de marzo de } 2001 . \text { La vivienda que ocupa la Casa de la } \\
\text { Cultura fue construida en } 1924 \text { por don Juan Bautista Matamoros, } \mathrm{k} \\
\text { ebanista y constructor de gran renombre en su época. }\end{array}$ \\
\hline
\end{tabular}

\section{Propuesta}

Esta propuesta se estructura en dos partes: primero, se desarrolla la ruta turística arquitectónica dentro del cantón esparzano, para, luego, analizar la factibilidad de complementar la ruta propuesta con la asignatura de Educación Cívica del MEP.

\section{Ruta de histórico-arquitectónica}

Dentro de los puntos más importantes por considerar, a la hora de elaborar la ruta, tenemos los siguientes:

Atractivos arquitectónicos: los 8 monumentos de importancia histórico-arquitectónica antes descritos (Cuadro 1).

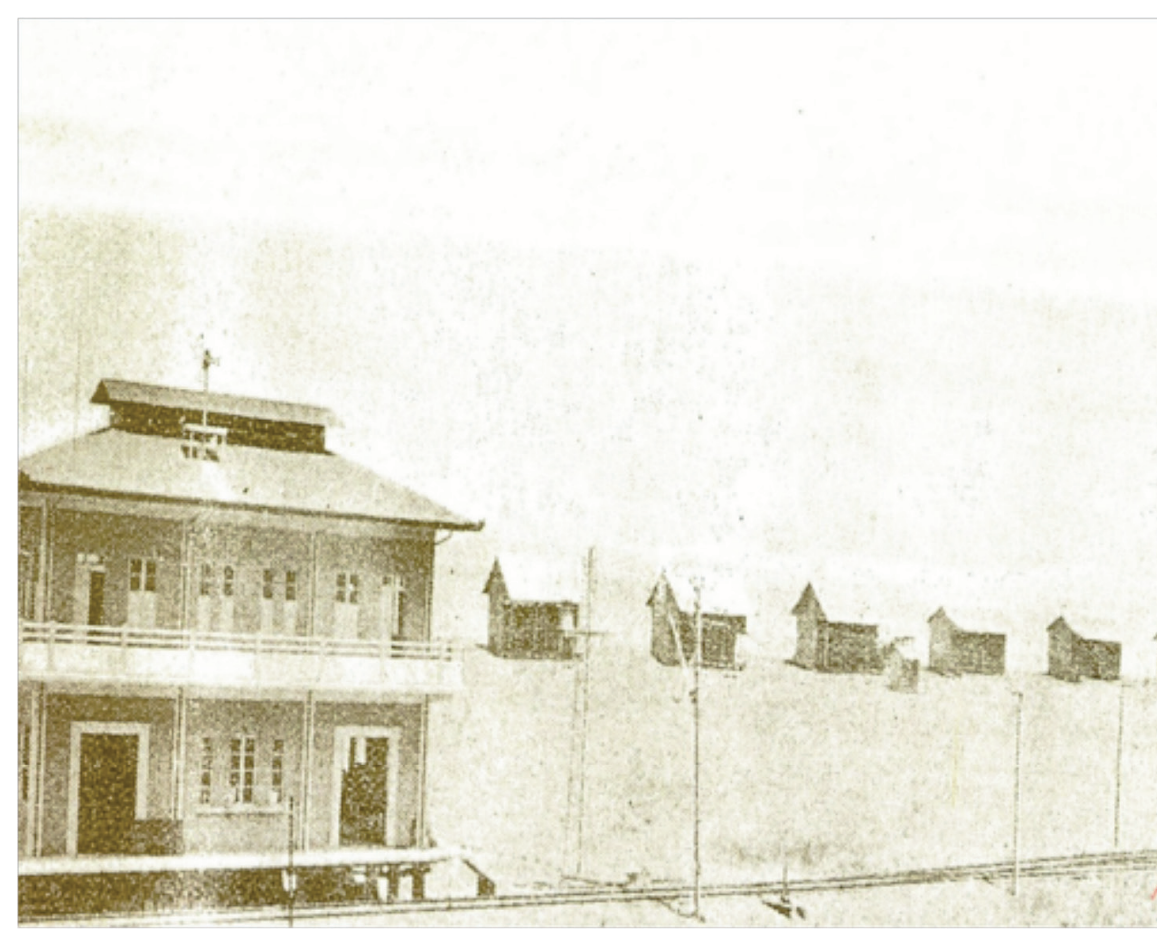

Figura 2. La estación de Caldera y el campamento de 1940 para los peones. Fuente: Marcos Hernán Elizondo. 
- Público meta: estudiantes de $8 .^{\circ}$ grado de educación secundaria.

- Cantidad de actividades: en el recorrido se realizarán 8 actividades (una por cada lugar visitado).

- Duración de la ruta: un día.

- Requerimientos de servicios: trasporte y alimentación.

Con base en la información citada, se presenta la siguiente propuesta que se denominará como "Arquiesparza", aludiendo al lugar y a su arquitectura.

\section{Cuadro 2}

\section{Ruta histórico-arquitectónica: "Arquiesparza"}

\begin{tabular}{|c|c|c|}
\hline Día & Hora & Actividad \\
\hline $\begin{array}{l}1 \\
\text { Ruta turística- } \\
\text { arquitectónica: } \\
\text { "Arquiesparza" }\end{array}$ & 7:30 a.m. & Salida de la institución educativa. \\
\hline & 8:00 a.m. & Llegada al primer monumento: puente Las Damas. \\
\hline & $8: 10$ a.m. & $\begin{array}{l}\text { Recorrido por el monumento y charla imparti- } \\
\text { da por el profesor o grupo de estudiantes. }\end{array}$ \\
\hline & 9:00 a.m. & Se retoma el tour hacia el monumento: túnel de Cambalache. \\
\hline & 9:10 a.m. & $\begin{array}{l}\text { Recorrido por el monumento y charla imparti- } \\
\text { da por el profesor o grupo de estudiantes. }\end{array}$ \\
\hline & 10:00 a.m. & $\begin{array}{l}\text { Se desplazan hacia el siguiente monu- } \\
\text { mento: el túnel de Miraflores. }\end{array}$ \\
\hline & $10: 10$ a.m. & $\begin{array}{l}\text { Recorrido por el monumento y charla imparti- } \\
\text { da por el profesor o grupo de estudiantes. }\end{array}$ \\
\hline & 11:00 a.m. & Se visita el siguiente lugar: la Estación de Caldera. \\
\hline & 11.10 a.m. & $\begin{array}{l}\text { Tour dentro de las instalaciones para apreciar su belleza y } \\
\text { charla impartida por el profesor o grupo de estudiantes. }\end{array}$ \\
\hline & $12 \mathrm{md}$. & Almuerzo en restaurante local. \\
\hline & 1.00 p.m. & Regreso al centro de Esparza y visita al cementerio central. \\
\hline & 1.10 p.m. & $\begin{array}{l}\text { Recorrido por los pasillos del cementerio para } \\
\text { apreciar las esculturas y charla impartida por } \\
\text { el profesor o grupo de estudiantes. }\end{array}$ \\
\hline
\end{tabular}




\begin{tabular}{|c|c|c|}
\hline Día & Hora & Actividad \\
\hline $\begin{array}{l}1 \\
\text { Ruta turística- } \\
\text { arquitectónica: } \\
\text { "Arquiesparza" }\end{array}$ & 2.00 p.m. & Se realiza la visita a la Casa de la Cultura. \\
\hline & 2:10 p.m. & $\begin{array}{l}\text { Se coordina con la Asociación para el Rescate y } \\
\text { Desarrollo de la Cultura para una charla educa- } \\
\text { tiva y datos de interés sobre el inmueble. }\end{array}$ \\
\hline & 3:00 p.m. & Se realiza la visita a la escuela Arturo Torres Martínez. \\
\hline & 3:10 p.m. & $\begin{array}{l}\text { Se recorren las instalaciones del inmueble y charla im- } \\
\text { partida por el profesor o grupo de estudiantes. }\end{array}$ \\
\hline & 4:00 p.m. & Se visita el último sitio de la ruta: parque Ignacio Pérez. \\
\hline & 4:10 p.m. & $\begin{array}{l}\text { Se realiza un recorrido por el parque y se realiza una charla } \\
\text { impartida por el profesor o grupo de estudiantes en el quiosco. }\end{array}$ \\
\hline & 5:00 p.m. & $\begin{array}{l}\text { Se agradece la participación de los estudiantes y } \\
\text { se da por finalizada la ruta "Arquiesparza". }\end{array}$ \\
\hline
\end{tabular}

La ruta turística "Arquiesparza" antes propuesta presenta las siguientes características:

1. Está diseñada para ser utilizada por el docente a cargo de la clase.

2. El docente debe preparar material de apoyo sobre los atractivos turísticos, con el fin de enriquecer la visita.

3. Los sitios más alejados del centro de Esparza son visitados en el transcurso de la mañana (necesitan trasporte). Los que se encuentran relativamente cerca del centro de la ciudad se visitan en la tarde (se pueden realizar a pie).

4. Presenta flexibilidad para involucrar pequeñas exposiciones a cargo de grupos de estudiantes.

5. Con una adecuada coordinación se pueden solicitar charlas educativas por parte de terceros, cuando el inmueble lo permita (escuela Arturo Torres, la Casa de la Cultura).

6. Los estudiantes disponen de tiempo para explorar el sitio y formar sus propios criterios.

\section{La ruta "Arquiesparza" propuesta como técnica didáctica en la formación cívica del MEP}

Actualmente, el Ministerio de Educación Pública (MEP) imparte, dentro de las materias curriculares, la asignatura de Educación Cívica, la cual es de carácter obligatorio para todos los estudiantes de secundaria, desde el séptimo grado hasta el undécimo año lectivo. 
Entonces, ¿qué se debe entender por Educación Cívica dentro del ámbito educativo? Para solventar esta duda, Castro (1996, p. 7) expone que: "Esta disciplina procura la formación del ciudadano que la sociedad costarricense demanda: un ciudadano comprometido con los valores, costumbres, tradiciones y con el sistema democrático de vida, así como con la familia y la comunidad". En la definición anterior, se puede notar la importancia de la implementación de esta materia en el sistema educativo costarricense.

Siguiendo estos lineamientos, la presente propuesta tiene como objetivo utilizar una ruta histórico-arquitectónica ("Arquiesparza") para complementar la formación cívica de los estudiantes de secundaria. No obstante, se debe aclarar que el grado más pertinente para la implementación de la "ruta cultural" es el $8^{\circ}$. año: pues la temática que se desarrolla en el primer trimestre denominada "Las personas jóvenes reivindicamos el sentido de identidad" es el título que abarca varios temas como: la historia y cultura de la comunidad, el patrimonio cultural tangible e intangible y el fortalecimiento de la identidad y cultura, entre otros. (MEP, 2009).

Sin embargo, para responder a la pregunta: ¿cómo incluir la ruta "Arquiesparza" en la metodología por utilizar por parte del docente?, se deben analizar algunas variables que proporcionen sustento a la propuesta, entre estas se encuentran:

\section{Importancia o relevancia de la ruta turística}

La importancia de esta propuesta radica en que los estudiantes puedan generar conceptos nuevos a partir de vivencias personales al experimentar e interactuar con los atractivos culturales. Además, el docente debe generar las oportunidades para que los estudiantes creen nuevos conocimientos que funcionen como base para su formación cívica.

Para que esta "ruta" tenga éxito en su objetivo, es necesario que el docente que pretenda utilizar esta estrategia metodológica, que no incurra en el error de enfocar la ruta turística "Arquiesparza" como algo netamente histórico. Claro está que este es un aspecto implícito, pero no debe ser el factor dominante, por lo cual se propone la utilización de diferentes técnicas didácticas para hacer de esta una experiencia amena.

\section{Viabilidad}

Desde el punto de vista académico es viable utilizar esta propuesta didáctica en la asignatura de Educación Cívica. La inquietud que se presenta radica en poder realizar una gira académica con estudiantes de secundaria, lo cual es permitido por el MEP. Para esto, se puede utilizar la modalidad de "excursión o visita a la empresa" la cual es definida por el MEP (2010, p. 29) como:

Su finalidad responde a una temática en particular, por ejemplo visitar 2 o 3 lugares para una charla, una exposición, presentación de un profesional. Su salida responde a un día de clase. Tanto docentes como estudiantes deben presentar un informe de la salida.

Esta modalidad se ajusta a nuestra metodología, ya que la ruta está diseñada para realizarse en un día. 


\section{Evaluación}

Esta es la parte más importante y la de mayor complejidad; pero, al mismo tiempo, es la que permite desarrollar diferentes estrategias educativas. También es un factor decisivo que justifica la implementación de esta "ruta cultural" en la metodología utilizada por los educadores.

Es importante mencionar que todo sistema educativo necesita una herramienta que permita medir el grado de asimilación de los conocimientos por parte de los estudiantes. Para este fin, el MEP ha desarrollado una evaluación que consta de un total equivalente al $100 \%$, el cual es dividido en diferentes rubros con sus respectivos porcentajes. (MEP, 2009).

Para el caso específico de la materia de Educación Cívica se presenta un cambio bastante importante en la distribución de los porcentajes, donde sobresale la disminución del porcentaje en $8 .^{\circ}$ año del rubro "pruebas", el cual correspondía al $65 \%$ del porcentaje total, a tan solo $30 \%$; el restante $35 \%$ se le acredita a un nuevo rubro denominado "Proyecto". (MEP, 2009).

\section{Cuadro 3}

\section{Distribución porcentual del rubro "Proyecto"}

\begin{tabular}{|l|c|}
\hline Proyecto & $\mathbf{3 5 \%}$ \\
\hline Participación activa durante la ruta cultural. & $5 \%$ \\
\hline $\begin{array}{l}\text { Pequeña exposición de la reseña históri- } \\
\text { ca de los sitios a visitar en subgrupos. }\end{array}$ & $10 \%$ \\
\hline Trabajo de investigación o ensayo. & $20 \%$ \\
\hline Fuente: Elaboración propia & \\
\hline
\end{tabular}

Siguiendo los parámetros establecidos por el MEP, la propuesta solo sería viable si es evaluada dentro del rubro "Proyecto," lo que le proporcionaría un valor porcentual de $35 \%$ dentro de la nota final del trimestre.

Lo interesante de esta evaluación es su flexibilidad, ya que cada docente puede proponer una forma de distribuir ese $35 \%$ del porcentaje en subcategorías, para esta propuesta se sugiere la siguiente distribución:

Siguiendo la propuesta anterior, debe hacerse hincapié en el hecho de que su éxito ayude a la formación cívica de los participantes. Para esto, el trabajo de investigación no debe ir orientado a la connotación histórica de los atractivos arquitectónicos visitados, sino, más bien, a proponer trabajos académicos, individuales o grupales, que giren en torno su situación actual, que procuren solventar alguno de los problemas que estos presentan, por ejemplo:

- Falta de conocimiento de los monumentos histórico-arquitectónicos por parte de las personas del cantón y del país.

- Desconocimiento de la declaratoria de su interés histórico-arquitectónico. 
- El papel que deben desempeñar las instituciones del Estado en pro de los monumentos y su conservación, entre otros.

El docente puede utilizar su creatividad para proponer una serie de proyectos orientados a resolver los problemas antes citados, por ejemplo:

- Elaboración de una revista, física o digital, informativa para la comunidad.

- $\quad$ Propuestas, para instituciones, para una adecuada gestión de los monumentos.

- Elaboración de un mural informativo para el colegio y/o municipalidad del cantón.

- Elaboración de ensayos orientados al rescate del patrimonio arquitectónico del lugar y su relevancia en la formación de la identidad del ser esparzano, entre otros.

De esta forma se estaría cumpliendo con los objetivos propuestos, ya que la ruta "turística arquitectónica" permitiría que los estudiantes se enfrenten a situaciones que afectan directamente a su comunidad e identidad nacional, mejorando los aprendizajes impuestos por la materia de Educación Cívica.

\section{Reflexiones finales}

La estrategia propuesta se centra en el cantón de Esparza; sin embargo, no se debería asumir que este tipo de proyectos solo pueden desarrollarse en este sector del país; más aun, deben ser considerados como un ejemplo por seguir que podría promover el desarrollo de nuevas rutas culturales que ayuden a la formación cívica del estudiantado, tomando en cuenta que la pertinencia de este tipo de propuestas cumplen con los lineamientos del MEP, aplicados a cualquier centro educativo de secundaria.

El rol del educador es el más importante dentro de la propuesta, ya que es este quien debe guiar tanto en la ruta cultural, como en el proceso de aprendizaje; por lo cual debe estar comprometido con la logística y la preparación de los materiales de apoyo.

Este tipo de proyectos - aunque se desarrollen desde un centro educativo- involucran una gran cantidad de actores sociales, como las familias, la comunidad y las instituciones, los cuales realizarán aportes importantes; pero, al mismo tiempo, adquirirán valiosos aprendizajes de la Educación Cívica.

Los trabajos académicos que surjan de la propuesta didáctica, y según el criterio del docente, deben ser orientados a cumplir con su finalidad y no quedar en el papel como una simple idea.

Por último, debemos tomar en cuenta que esta es una propuesta didáctica que no se ha llevado a la práctica, por lo cual es necesario mencionar que para el docente, con intenciones de incorporarla dentro de su metodología, sería sumamente importante realizar algún control para medir su efectividad o para realizar los cambios necesarios y así fortalecer los aprendizajes. Una buena opción es aplicar un cuestionario o grupo focal con los estudiantes. 


\section{Bibliografía}

Alfaro, W., Porras, J. (2006). Patrimonio cultural en el cantón de Turrialba: diseño de un circuito turístico cultural. (Tesis inédita de licenciatura). Universidad de Costa Rica, sede Guanacaste.

Castro, M. (1996). Educación cívica 7, 1. ed. San José: Ediciones FARBEN.

Lagos, José Ángel. (22 de junio de 1976). "Esparza o Esparta". La Nación. Recuperado de: http://news.google.com/newspapers/p/lanacion?id=AkIfAAAAIBAJ\&sjid $=$ CdEEAAAAIBAJ\&pg=1370,4563917\&dq=ESPARTA\&hl=en

"Gran baile social en Esparta". La Nación. (01 de febrero de 1965). Recuperado de: http://news.google.com/newspapers/p/lanacion?id=bhwiAAAAIBAJ\&sjid=f3oE AAAAIBAJ\&pg=4859,3633276\&dq=ESPARTA\&hl=en

"Regio té de modas en Esparta". La Nación. (13 de agosto de 1966). Recuperado de: http://news.google.com/newspapers/p/lanacion?id=K_UcAAAAIBAJ\&sjid=THoE AAAAIBAJ \&pg $=3352,7806552 \& d q=E S P A R T A \& h l=e n$

Ministerio de Cultura, Juventud y Deportes. "Cantón 02 Esparza". Recuperado de: http://www.mcj.go.cr/temas_artes/patrimonio/cicpc/inmueblespatrimoniales/ puntarenas/esparza/canton_\%2002_esparza.aspx

Ministerio de Educación Pública. (2009). Programas de estudios Educación Cívica: tercer ciclo de educación general básica y educación diversificada. San José. Recuperado de http://www.mep.go.cr/sites/default/files/programadeestudio/ programas/civica3cicloydiversificada.pdf

Ministerio de Educación Pública. (2010). Especialidad turismo ecológico: diseño curricular bajo el modelo de educación basada en normas por competencia. San José. Recuperado de http://www.mep.go.cr/sites/default/files/descargas/programas-de-estudio/turismoecologico11.pdf

Municipalidad de Esparza. (2014). "Patrimonios del cantón de Esparza" Recuperado de: http://www.muniesparza.go.cr/index.php/quienes-somos/patrimonios

Sandi, Gerardo. (2003). "Reseña Histórica: Puntarenas-Esparza y Montes de Oro". Revista Acontecer. Puntarenas, Costa Rica. 
